\title{
O suicídio no Estado do Tocantins
}

\author{
The suicide in the State of Tocantins \\ El suicidio en el Estado del Tocantins
}

Erivaldo da Silva Soares Filho ${ }^{1}$, Ludmilla Carolinne Santana Correia', Patricia Rogalski Lima', Helierson Gomes ${ }^{2 *}$, Andrielly Gomes de Jesus².

\section{RESUMO}

Objetivo: Avaliar o perfil e situação epidemiológica na incidência dos casos de suicídios no estado do Tocantins no período de 2005 - 2015. Métodos: Estudo de caráter epidemiológico dos casos de suicídio no estado do Tocantins, com base em métodos descritivo e ecológico, tendo as regionais de saúde do estado como unidades de observação. Os dados epidemiológicos estudados serão os índices de mortalidade por suicídio classificados por local/cidade de residência, regional de saúde, ano de notificação, sexo, raça/cor, faixa etária e grau de escolaridade. Resultados: $O$ presente estudo mostra em seus resultados taxas elevadas de suicídio na população jovem adulta, solteiros, idosos, destacando a população masculina, por sua incidência ser três vezes maior em relação à feminina, e quanto ao grau de escolaridade, seu maior quantitativo está relacionado àqueles que possuem até sete anos de estudo. Considerações Finais: Por ser um problema de saúde pública, faz-se necessário promover estratégias para que os altos índices sejam diminuídos, onde diversos fatores estão relacionados ao chamado comportamento suicida, fatores estes como socioeconômicos, biológicos e transtornos mentais. Através do conhecimento do perfil epidemiológico do suicídio do estado é possível traçar estratégias de prevenção e intervenções voltadas aos fatores de risco.

Palavras - chave: Suicídio, Epidemiologia, Tocantins.

\begin{abstract}
Objective: To evaluate the profile and the epidemiological situation in the incidence of suicide cases in the state of Tocantins in the period 2005 - 2015. Methods: Epidemiological case studies in the State of Tocantins, based on descriptive and ecological methods. units of observation. The epidemiological data will be the suicide mortality indexes classified by place of residence, health region, year of notification, sex, race / color, age group and schooling level. Results: The present study shows high suicide rates in the young adult population, singles, elderly, highlighting the male population, due to its incidence being three times higher in relation to the female, and the highest quantitative related to those who have up to seven years of study. Final Considerations: Because it is a public health problem, it is necessary to promote strategies so that high rates are reduced, where several factors are related to the so-called suicidal behavior, such as socioeconomic, biological and mental disorders. Through the knowledge of the epidemiological profile of state suicide, it is possible to draw up prevention strategies and interventions directed at risk factors.
\end{abstract}

Key words: Suicide, Epidemiology, Tocantins.

${ }^{1}$ UNIESP S.A - Faculdade de Colinas do Tocantins - TO. Tocantins - TO.

2Universidade Federal do Tocantins - UFT, Araguaína/Tocantins. *E-mail: profhelierson@gmail.com

SUBMETIDO EM: 4/2019

ACEITO EM: 5/2019

PUBLICADO EM: 7/2019

REAS/EJCH | Vol. 11(12) | e712 | DOI: https://doi.org/10.25248/reas.e712.2019 Página 1 de 9 


\section{RESUMEN}

Objetivo: Evaluar el perfil y situación epidemiológica en la incidencia de los casos de suicidios en el estado de Tocantins en el período de 2005 a 2015. Métodos: Estudio de carácter epidemiológico de los casos de suicidio en el estado de Tocantins, con base en métodos descriptivos y ecológicos, teniendo las regiones de salud del estado como unidades de observación. Los datos epidemiológicos estudiados serán los índices de mortalidad por suicidio clasificados por local / ciudad de residencia, regional de salud, año de notificación, sexo, raza / color, grupo de edad y grado de escolaridad. Resultados: El presente estudio muestra en sus resultados tasas elevadas de suicidio en la población joven adulta, solteros, ancianos, destacando la población masculina, por su incidencia ser tres veces mayor en relación a la femenina, y en cuanto al grado de escolaridad, su mayor cuantitativo está relacionado con aquellos que tienen hasta siete años de estudio. Consideraciones finales: Por ser un problema de salud pública, se hace necesario promover estrategias para que los altos índices sean disminuidos, donde diversos factores están relacionados al llamado comportamiento suicida, factores tales como socioeconómicos, biológicos y trastornos mentales. A través del conocimiento del perfil epidemiológico del suicidio del estado es posible trazar estrategias de prevención e intervenciones dirigidas a los factores de riesgo.

Palabras clave: Selbstmord, Epidemiologie, Tocantins.

\section{INTRODUÇÃO}

Atualmente o suicídio tem afetado indivíduos independentes de sua raça, sexo, faixa etária e classe econômica, sendo assim, o mesmo já representa um problema de saúde pública. O perfil do suicídio vem sofrendo alterações, passando da faixa etária mais idosa para a mais jovem (BOTEGA NJ, 2007; VIEIRA KFL, et al, 2010; SCHNITMAN G, et al, 2010).

Vieira KFL, et al, (2010), utiliza a definição clássica de Émile Durkheim para o suicídio, onde, trás que o ato de suicídio é quando a própria vítima tenta praticar qualquer meio que venha resultar em morte independentemente do método. suicídio é todo caso de morte que resulta, direta ou indiretamente, de um ato positivo ou negativo praticado pela própria vítima.

Vários fatores podem ser relacionados à tentativa de suicídio, tais como o desemprego, a pobreza, perda de entes queridos, rupturas de relações afetivas, problemas no trabalho e discussões familiares, estando estes em conformidade com diversos estudos acerca do tema (PACHECO JS, et al, 2010).

Vidal CEL, et al (2014), descreve que as mortes por suicídio em indivíduos do sexo masculino representam $80,1 \%$ do total de óbitos entre a faixa etária de 20 a 49 anos, já no sexo feminino a faixa etária mais acometida é entre 40 e 49 anos. Os homens utilizam métodos com alto grau de letalidade como, enforcamento, uso de arma de fogo e lugares elevados, sendo sua maior ocorrência proveniente de problemas socioeconômicos, incluindo questões profissionais e financeiras, enquanto as mulheres apresentam um maior quantitativo em tentativas de suicídio e menor quantitativo em óbitos, tendo como fatores o envolvimento em conflitos interpessoais afetivos com o companheiro ou familiares, utilizando como método a ingestão de medicamentos e outras substâncias tóxicas (PACHECO JS, et al, 2010).

O suicídio é um fenômeno complexo que ocorre em toda parte do mundo, é investigado em diversos campos científicos por meio de abordagens complementares e também antagônicas (SCHNITMAN G, et al, 2010).

O suicídio tornou-se objeto de estudo das mais diversas áreas, não somente das ciências exatas, biomédicas, humanas e sociais, mas também das artes e das religiões, pois tem causado grande desconforto à população em geral, passando a ser conhecida como "suicidologia". Esse fenômeno não é considerado um fator pessoal e sim social, como alguns autores renomados no assunto afirmam em suas pesquisas (ALMEIDA LN, et al, 2015). 
A integração social durkehimiana descreve três tipos de suicídios, sendo eles o suicídio altruísta: acontece quando uma pessoa encontra-se inteiramente ligada à sociedade, a ponto de tirar a própria vida para satisfazer outras pessoas; o suicídio egoísta: que ocorre devido o afastamento do indivíduo do meio social; e por fim o suicídio anômico: que acontece devido à ausência de normas e leis em uma sociedade, o que levaria o indivíduo a desequilibrar-se psicologicamente e ficar mais vulnerável a cometer o suicídio (ALMEIDA LC, et al, 2015).

Segundo a Organização Mundial da Saúde (OMS), 800 mil pessoas praticam o ato de suicídio todos os anos no mundo, ressalta também que cerca de $75 \%$ dos casos ocorrem nos países de menor poder econômico. Mundialmente, a mortalidade por suicídio aumentou de forma alarmante. Os índices com maiores incidências de suicídios ocorrem em países da Europa Oriental (Hungria, Finlândia), Japão, América Latina, países Muçulmanos e asiáticos (ABASSE MLF, et al, 2009; FIOCRUZ, 2014).

Dados da OMS mostram que no ano de 2000, aproximadamente 1 milhão de pessoas morreram por suicídio no mundo, o que representa 1 morte a cada 40 segundos (MACENTE LB, 2010). De acordo com Vidal CEL, (2013) no ano de 2020 terá uma projeção de mais de 1 milhão e meio de pessoas que poderão cometer o suicídio, a estimativa é que o número de tentativas seja até 20 vezes maiores que os números de mortes.

De acordo com estudos de Botega NJ, (2014), a cada 45 segundos ocorre 1 suicídio no mundo, na maioria dos casos de suicídio existe a presença de transtornos mentais, sendo estes considerados fatores de risco importantes, a situação de risco é agravada quando ocorre a combinação de depressão e alcoolismo, por exemplo, ou a combinação simultânea de mais de um transtorno mental como, ansiedade e agitação. Segundo Fonseca DL, et al, (2010), situações estressantes como morte de pessoas íntimas, separação conjugal, desemprego, dentre outros fatores, podem propiciar transtornos mentais ou agravar os já existentes.

No Brasil até pouco tempo o suicídio não era visto como um problema de saúde pública, porém nos dias atuais devido à elevação de suas taxas, o mesmo já passou a ser considerado, estando localizado entre os 10 países com números absolutos em casos de suicídios, chegando até 6 mil casos por ano na população em geral. Visto que a sua taxa de mortalidade por suicídio é estimada em 4,1 por 100 mil para a população total (ARAÚJO LC, et al, 2010; SCHNITMAN G, et al, 2010).

O suicídio no Brasil não é considerado um crime e sim uma conduta antijurídica, ocupando a 8ª posição no ranking mundial, onde sua maior predominância é em jovens e adolescentes. Existe uma maior concentração de suicídios na região Sul - Rio Grande do Sul e na região Centro Oeste - Mato Grosso do Sul. Havendo ainda um grande aumento nos números de casos na região Norte, destacando-se, entre eles, estados do Tocantins, Amapá e Acre (FERREIRA NS, et al, 2014; ALMEIDA LN, et al, 2015).

O suicídio constitui uma importante questão de saúde pública no mundo inteiro. Estudos realizados mostram que a temática ainda é pouco estudada em algumas regiões do Brasil, em especial na região Norte, que juntamente com a região Centro-Oeste apresenta, além de uma literatura bastante resumida sobre 0 tema, fragilidade nas informações sobre mortalidade (LOVISI GM, et al, 2009; FERREIRA NS, et al, 2014).

O estado do Tocantins ocupava a 19ª colocação em meados de 1998, já em 2006 houve um grande índice de suicídio e passou a ocupar o 6 o lugar. Dentre todas as capitais brasileiras, a capital do estado do Tocantins está entre as primeiras colocadas em casos de suicídios (FERREIRA NS, et al, 2014).

Os transtornos mentais estão entre os fatores de risco mais importantes para o comportamento suicida, estando presente em mais de $90 \%$ daqueles que cometem suicídio. Dentre os problemas de nível psíquico a depressão ganha destaque, pois se encontra cada vez mais presente, sendo considerado um fator de alto risco, além da depressão, problemas como, ansiedade, estresse, solidão, esquizofrenia, dentre outras, agravam a situação (ABREU KP, et al, 2010). Estudos de Santos AS, et al, (2009), também destacam a presença da depressão variando de $13 \%$ a $53,8 \%$ dos casos.

No processo de implementação da Política de Saúde Mental no Brasil, a partir da publicação da Lei no 10.216 dispondo sobre um novo modelo assistencial de proteção e direito às pessoas portadoras de 
transtorno mental, pôde ser observado um aumento progressivo de uma assistência em saúde mental extra hospitalar, de base comunitária, proporcionando aos portadores a oportunidade de serem reinseridos na comunidade e no seio familiar (OLIVEIRA EFA, 2011).

Diante disto, torna-se indispensável a realização de estudos sobre o respectivo tema abordado, para que seja possível contribuir de forma científica, identificando a dimensão e real situação do problema para oferecer informações que irão auxiliar na elaboração de programas de prevenção e enfretamento do problema (SCHNITMAN G, et al, 2010). Corroborando com este pressuposto esta pesquisa vem com o objetivo de avaliar o perfil e situação epidemiológica da incidência dos casos de suicídios no estado do Tocantins no período de 2005 a 2015.

\section{MÉTODOS}

O estudo realizado no estado do Tocantins, este localizado na região norte, conta com uma população estimada de 1.383 .445 habitantes e área de 277.720,567 km², um total de 139 municípios totalizado em uma densidade demográfica de 4,98 hab/km².

É subdividido em 8 microrregiões e 2 mesorregiões, seu Índice de desenvolvimento humano médio (IDH) é de 0,699 ocupando a $14^{\circ}$ colocação dentre as 27 unidades da federação (IBGE, 2017).

O estudo epidemiológico dos casos de suicídio no estado do Tocantins foi realizado com base em métodos descritivo e ecológico, tendo as regionais de saúde do estado como unidades de observação. $O$ estudo epidemiológico contemplou $100 \%$ das notificações de casos confirmados de suicídio nos municípios do estado do Tocantins e registrado na base de dados do Sistema de Informações de Vigilância Epidemiológica através da Ficha de Notificação de suicídio no período de 2005 a 2015.

Os dados epidemiológicos estudados foram os índices de mortalidade por suicídio classificados por local/cidade de residência, regional de saúde, ano de notificação, sexo, raça/cor, faixa etária e grau de escolaridade.

O banco de dados utilizado para a caracterização da Análise da Distribuição Espaço-Temporal dos casos foi construído a partir das bases de dados do Sistema de informação de agravos notificados (SINAN) na Secretaria de Saúde Pública do estado do Tocantins.

Para análise foi utilizado o programa Microsoft ${ }^{\circledR}$ Excel® 2008 for Mac, versão 12.2.8, para a depuração dos dados epidemiológicos e análise descritiva da incidência dos casos de suicídio. Dentre as variáveis foi realizado a análise de variação (ANOVA) e o teste de correlação de Pearson para analisar o grau de relação entre o nível de escolaridade com os casos de suicídio confirmados.

\section{RESULTADOS}

É possível identificar nas variáveis estudadas que a população jovem adulta (20 - 29 anos) do sexo masculino, destacando-se as três maiores regiões de saúde como: Araguaína - TO, Gurupi - TO e Porto Nacional - TO obtiveram maior predominância em casos absolutos de suicídios.

A variável faixa etária chama atenção por ter um menor quantitativo de casos absolutos em suicídio na população idosa (70 - 79 anos), porém, maior incidência chegando aos (126,9/100 mil/hab), um dado inferior à população jovem adulta. Os casos consumados de suicídio na população masculina chegam a ser três vezes maiores em relação à feminina, embora as mulheres apresentem maiores números de tentativas de suicídio (Tabela 1).

Em relação aos métodos utilizados chama atenção o método por enforcamento devido seu alto quantitativo sendo quatro vezes a mais em relação aos outros métodos utilizados, como intoxicação por pesticidas, uso de arma de fogo, ingestão por produtos químicos e dentre outros. O sexo masculino predomina com seu alto quantitativo sendo $(79,2 \%)$ enquanto o sexo feminino é $(21,8 \%)$ não podendo deixar de ressaltar os casos de subnotificações (Figura 1). 
Tabela 1 - Casos de Suicídio por região de saúde Estado do Tocantins.

\begin{tabular}{|c|c|c|c|c|}
\hline Variáveis & $\mathbf{N}$ & $\%$ & In & Var \\
\hline \multicolumn{5}{|l|}{ Sexo } \\
\hline Masculino & 738 & 79.2 & 95.9 & \multirow{2}{*}{$P=0,003$} \\
\hline Feminino & 193 & 20.8 & 25.8 & \\
\hline \multicolumn{5}{|l|}{ Faixa Etária } \\
\hline $10-14$ & 14 & 1.5 & 9.4 & \multirow{9}{*}{$P=0,0001$} \\
\hline $15-19$ & 79 & 8.4 & 55.9 & \\
\hline $20-29$ & 257 & 27.6 & 93.9 & \\
\hline $30-39$ & 200 & 21.4 & 80.6 & \\
\hline $40-49$ & 159 & 17 & 89.2 & \\
\hline $50-59$ & 86 & 9.2 & 69.3 & \\
\hline $60-69$ & 65 & 6.9 & 86.1 & \\
\hline $70-79$ & 49 & 5.2 & 126.9 & \\
\hline$>80$ & 17 & 1.8 & 107.2 & \\
\hline \multicolumn{5}{|l|}{ Região de Saúde } \\
\hline Bico Papagaio - TO & 93 & 9.9 & 43.7 & \multirow{8}{*}{$P=0.001$} \\
\hline Araguaína - TO & 196 & 21.1 & 63 & \\
\hline Miracema - TO & 90 & 9.6 & 60 & \\
\hline Rio Formoso - TO & 98 & 10.5 & 77 & \\
\hline Gurupi - TO & 119 & 12.7 & 80 & \\
\hline Porto Nacional - TO & 228 & 24.4 & 59 & \\
\hline Jalapão - TO & 28 & 3.5 & 35 & \\
\hline Dianópolis - TO & 78 & 8.3 & 63 & \\
\hline
\end{tabular}

Fonte: SINAN, 2018. Legenda; N: números absolutos, IN: incidência 100mil/hab, Var: Coeficiente de Variação.

Figura 1 - Óbitos por suicídio segundo método utilizado e sexo.

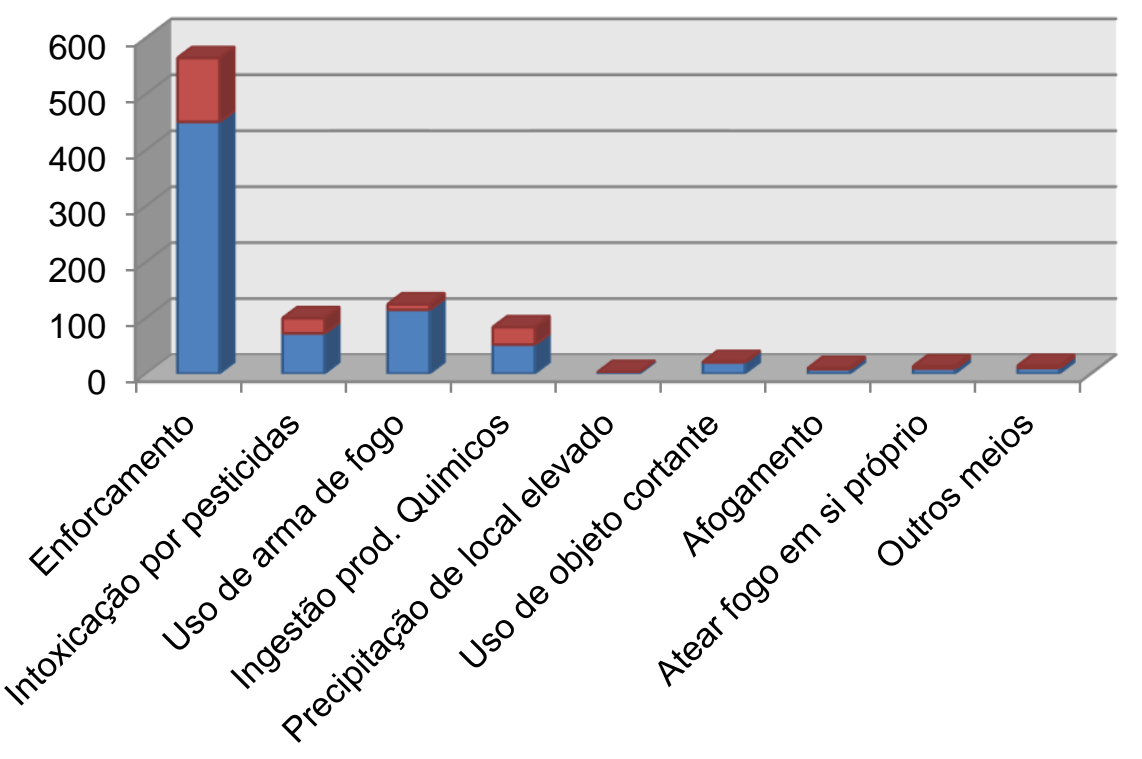

ufeminino $(21.8 \%)$

$\square$ masculino $(79.2 \%)$

Fonte: SINAN, 2018. 
Figura 2 - Percentual de mortes por suicídio segundo estado civil.

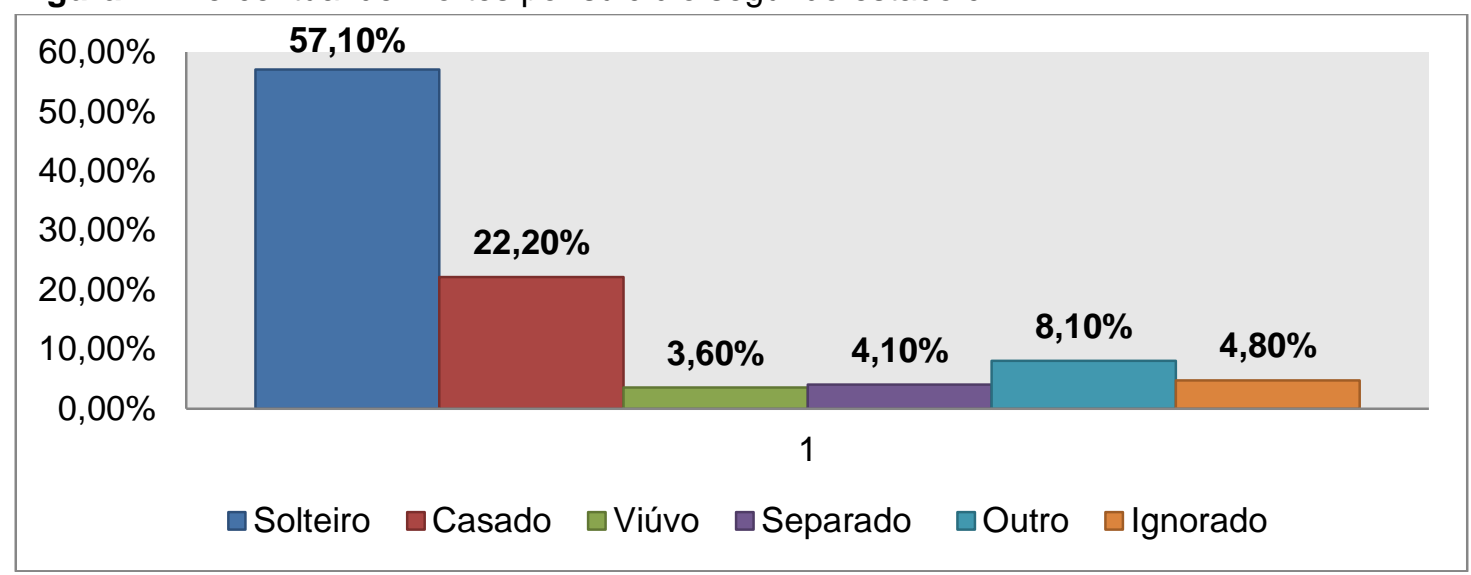

Fonte: SINAN, 2018.

A população solteira apresenta altas taxas de suicídio $(57,10 \%)$, sendo duas vezes maiores em relação aos casados $(22,20 \%)$, seguido pelos separados $(4,10 \%)$ e viúvos $(3,60 \%)$, constituindo um fator importante neste cenário, pois o suicídio é um problema multicausal (Figura 2).

Em relação ao grau de escolaridade, aproximadamente $60 \%$ dos casos é referente ao público com até sete anos de estudos, ou seja, não possuem o primeiro grau completo, constatou-se ainda uma relação negativa moderada entre o grau de escolaridade e os casos de suicídio $(r=-0,2)$, significando que conforme aumenta o nível de escolaridade há uma redução nos índices de suicídio da população (Figura 3).

Figura 3 - Percentual dos casos de suicídio segundo a escolaridade. Análise de relação de Pearson dos casos de suicídio com grau de escolaridade

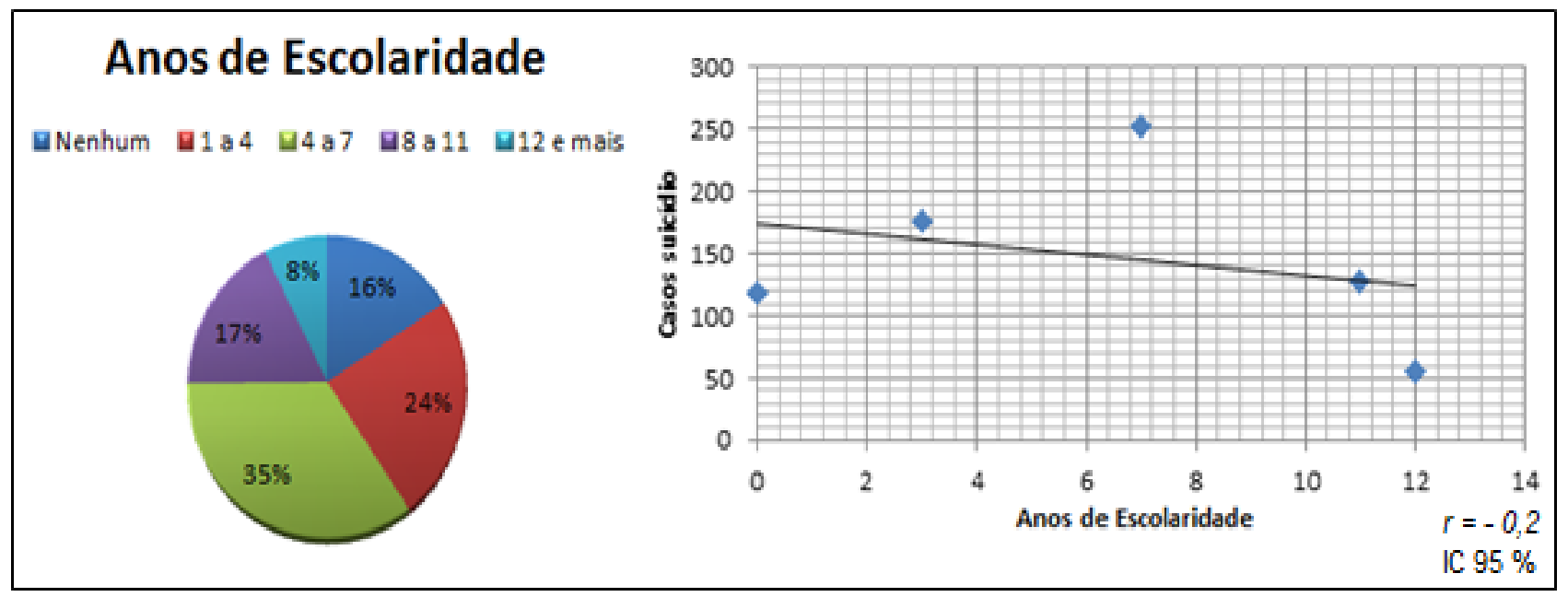

Fonte: Sinan, 2018.

\section{DISCUSSÃO}

Anualmente estima-se que 800 mil pessoas morrem por suicídio e para cada adulto que consuma o fato outros 20 tentam contra a própria vida, sendo considerado um fenômeno mundial, ainda que possa ser evitado, porém, ainda há um estigma muito forte o que impede a busca por ajuda. Diante do atual cenário, é de suma importância desenvolver ações baseadas em dados seguros, conhecer o perfil da população e principais causas. Surge então a necessidade de uma rede de atenção qualificada à saúde que garanta ações de vigilância, estratégias de enfrentamento e a garantia de uma linha de cuidados integrais no manejo dos casos de tentativas de suicídio melhorar o acesso e o acompanhamento dos usuários (FERNANDES DAA, 2016; BRASIL 2017). 
A maior taxa de suicídio se encontra na população masculina (72,9\%), em mulheres são maiores os números de tentativas e menores os números de casos consumados $(21,8 \%)$, este fato está relacionado aos homens terem maior acesso à armas de fogo e tecnologias letais, ao comportamento impulsivo e competitivo, maior sensibilidade aos fatores estressantes como as instabilidades financeiras, além da associação ao uso de álcool que diminui o autocontrole e favorece as condutas autodestrutivas, já as mulheres apresentam atitudes mais flexíveis, menor prevalência de alcoolismo, religiosidade e o fato de buscarem apoio nos momentos de crise, além de escolherem métodos menos letais como, ingestão de produtos químicos e pesticidas (SCHNITMAN G, et al, 2010; BOTEGA NJ, 2014; VIDAL CEL, et al, 2014).

Relacionado aos métodos mais utilizados no ato do suicídio especificamente no Estado do Tocantins encontra-se em conformidade com estudos de Abreu KP, et al, (2010), Vidal (2013) e Schnitman G, et al, (2010) que apresentam resultados semelhantes, fato este que pode ser atribuído aos homens utilizarem métodos mais letais como o uso de armas de fogo e enforcamento, método esse mais viável e de fácil acesso em qualquer ambiente familiar.

A variável faixa etária chama atenção devido sua alta taxa de incidência, sendo a população mais acometida pessoas com > de 75 aos de idade, fato encontrado não somente no Brasil mais também em outros países como Nova Zelândia e Japão, estes apresentam media de variação entre as idades de 25 a 64 anos, os dados são preocupantes visto que a previsão para 2030 é que o Brasil tenha a quinta população mais idosa do mundo chegando a ultrapassar o total de crianças entre zero e quatorze anos(BERTOLOTE JM, et al, 2010).

Contudo os dados de Bertolote JM, et al, (2010), confirmam que maiores taxas de incidência de suicídio são habitualmente encontradas entre pessoas idosas ( $>75$ anos), entretanto, em determinados países a situação é radicalmente inversa, como por exemplo, na Nova Zelândia, varia entre ( 25 e 34 anos), e no Japão, entre (55 e 64 anos).

Os índices brasileiros em casos de suicídios vêm passando por uma tendência de crescimento contínuo nos últimos 20 anos, sua taxa de mortalidade por suicídio encontra-se entre 4 e 5 óbitos por 100 mil habitantes. O estado do Tocantins em 1998 ocupava a 19a colocação em casos de suicídio, passou por um período de

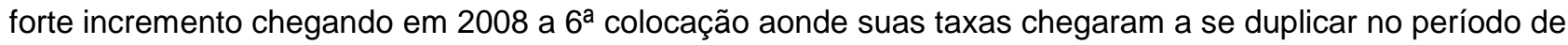
10 anos. Já nos últimos 9 anos, o suicídio e as tentativas passaram a representar uma grande preocupação para as cidades do estado, pelos seus elevados índices e por atingir principalmente a população mais jovem de 20 a 29 anos do sexo masculino (FERREIRA NS, et al, 2014; VIDAL CEL, et al, 2014).

Estudos de Schlösser A, (2014), apontam que o principal fator associado ao suicídio ou ideias suicidas na população jovem masculina está relacionado com a depressão, devido as dificuldades no enfrentamento das frustações pessoais adquiridas por meio disfunções familiares, abuso sexual, maus tratos e a insatisfação de suas necessidades básicas.

Em relação às regiões de saúde do Tocantins, três cidades chamaram atenção pelos seus elevados índices, sendo elas, Porto Nacional - TO chegando a (24, 4\%), Araguaína - TO com (21,1\%), e Gurupi - TO com (12,7\%), já a região do Rio Formoso - TO, chama atenção por sua baixa porcentagem (10,5\%), porém, maior incidência (77) por 100 mil habitantes em casos de suicídios, sendo que as três regiões anteriores variam de (59 a 80) por 100 mil habitantes.

Indivíduos solteiros, separados e viúvos possuem maior probabilidade de risco para cometer o suicídio. Em relação ao estado civil o estudo está de acordo com diversas pesquisas que demonstram maior número de suicídio entre os solteiros como a de Vidal CEL, et al (2014) e Fonseca DL, et al (2014), supondo-se que os solteiros são mais vulneráveis aos abalos emocionais por não terem vínculos sentimentais, como os laços amorosos, já os casados teriam um menor número de suicídios devido ao casamento reforçado pelos filhos (CUNHA FA, et al, 2016; OLIVEIRA EFA, 2011). Outro ponto preocupante pouco discutido ou até mesmo negligenciado são os casos de suicídio silenciosos, principalmente entre homossexuais do sexo masculino, tem sido uma prática frequentemente pensada ou executada entre os que não conseguem se ajustar às normas impostas pela sociedade ou resistir a elas. Segundo Natarelli TRP, et al, (2015) a saúde dessa 
população é afetada pela homofobia, que provoca quadros e comportamentos que caracterizam sofrimento mental e interfere na adoção de comportamentos e hábitos de vida saudáveis levando esses adolescentes homossexuais a uma situação de extrema vulnerabilidade, e que de certa forma tendência um percentual significativo nos quantitativos de casos notificados em jovens solteiros do sexo masculino (PRATA V, 2016)

A variável grau de escolaridade expõe que o maior percentual de suicídios é em indivíduos de baixa escolaridade, estando em conformidade com os estudos de Machado (2014) onde argumenta que o nível educacional juntamente com outros fatores sociais definem o status econômico e social de um indivíduo, desta forma o baixo nível de estudo pode proporcionar preocupações e estresse, levando a pessoa a ter baixa autoestima e chegar a cometer o suicídio. No entanto, existem estudos que divergem desta afirmação, como o de Gonçalves LRC, et al (2011), afirmando que quanto maior for o nível de instrução, maior será o risco de suicídio, tendo como explicação para esta afirmação o fato de que o trabalho de pessoas com alta escolaridade pode ser mais estressante e exigentes deixando-as mais vulneráveis ao suicídio.

Considerando que o fenômeno do suicídio vem de forma agravante na saúde pública, que afeta a sociedade em todos seus aspectos e que pode ser prevenido com politicas públicas eficazes em todas as esferas governamentais, no inicio do ano de 2019 foi Instituída a Política Nacional de Prevenção da Automutilação e do Suicídio, Lei no 13.819/2019, tornando obrigatório a notificações compulsórias de forma sigilosa e rigorosa dentro de todos ambientes da saúde, segurança, escolas e conselhos tutelares. Além das notificações, vem fortalecer a criação de um sistema em nível nacional onde os estados municípios devem contribuir com a politica criando meios de comunicações gratuitos, promover a saúde mental, controlar os fatores determinantes e condicionantes saúde mental, dentre outros, tornando possível identificar a real situação de cada local e contribuindo para que medidas preventivas sejam realizadas (BRASIL, 2019).

\section{CONSIDERAÇÕES FINAIS}

O presente estudo mostra em seus resultados taxas elevadas de suicídio na população jovem adulta, solteiros, idosos, destacando a população masculina, por sua incidência ser três vezes maior em relação à feminina, e quanto ao grau de escolaridade, seu maior quantitativo está relacionado àqueles que possuem até sete anos de estudo. Por ser um problema de saúde pública, faz-se necessário promover estratégias para que os altos índices sejam diminuídos, sabe-se que é um problema de múltiplas causas e está associado a diversos fatores que estão relacionados ao comportamento suicida. Contudo, por meio do conhecimento do perfil epidemiológico do suicídio do estado é possível traçar estratégias de prevenção e intervenções voltadas aos fatores de risco. Portanto a pesquisa vem reforçar a necessidade de que sejam realizados mais estudos voltados ao tema, pois as mesmas são escassas na região norte do Brasil.

\section{REFERÊNCIAS}

1. ABASSE MLF, et al. Análise epidemiológica da morbimortalidade por suicídio entre adolescentes em Minas Gerais, Brasil. Ciênc. saúde coletiva vol.14 no.2 Rio de Janeiro Mar./Apr. 2009.

2. ABREU KP, et al. Comportamento suicida: fatores de risco e intervenções preventivas. Rev. Eletr. Enf. [Internet]. $2010 ; 12(1): 195-200$.

3. ALMEIDA LN, et al. O suicídio no Brasil: Um desafio às Ciências Sociais. REBELA, v.5, n.3. set./dez. 2015.

4. ARAÚJO LC, et alldeação suicida na adolescência: um enfoque psicossociológico no contexto do ensino médio. Psico-USF, v. 15, n. 1, p. 47-57, jan./abr. 2010.

5. BRASIL. 2019. Lei no 13.819, de 26 de abril de 2019. Disponível em < http://www.in.gov.br/web/dou/-/lein\%C2\%BA-13.819-de-26-de-abril-de-2019-85673796 >. Acesso em 31 de maio de 2019.

6. BRASIL. 2017. Ministério da Saúde. Boletim Epidemiológico Secretaria de Vigilância em Saúde Volume $48 N^{\circ} 30$ 2017.

7. BERTOLOTE JM, et al. Detecção do risco de suicídio nos serviços de emergência psiquiátrica. S87 • Revista Brasileira de Psiquiatria • vol $32 \cdot$ Supl II • out2010.

8. BOTEGA NJ, et al. Suicídio: saindo da sombra em direção a um Plano Nacional de Prevenção. Rev Bras Psiquiatr. $2007 ; 29(1): 7-8$ 
9. BOTEGA NJ. Comportamento suicida: epidemiologia. Departamento de Psicologia Médica e Psiquiatria da Faculdade de Ciências Médicas da Universidade Estadual de Campinas, Campinas, SP, Brasil. 2014 I volume 25 I número 3 I 231-236.

10. CUNHA FA, et al. Análise documental sobre os suicídios ocorridos na região de jundiaí entre 2004 e 2014 . Red de Revistas Científicas de América Latina y el Caribe, España y Portugal. SALUD \& SOCIEDAD V. 7|No. 2 | MAYO AGOSTO | 2016.

11. FERREIRA NS, et al. Fatores de risco relacionados com suicídios em Palmas (TO), Brasil, 2006-2009, investigados por meio de autópsia psicossocial. Ciência \& Saúde Coletiva, 19 (1):115-126, 2014.

12. FERANDES DAA, et al. Perfil epidemiológico das tentativas de suicídio em Palmas-Tocantins, de 2010 a 2014. Tempus, actas de saúde colet, Brasília, 10(4), 09-23, dez, 2016.

13. FONSECA DL, et al. Apoio social, E eventos estressantes e depressão em casos de tentativa de suicídio: um estudo de caso-controle realizado em um hospital de emergência do Rio de Janeiro. Cad. Saúde Colet., 2010, Rio de Janeiro, 18 (2): 217-28.

14. FRIOCRUZ. Fundação Oswaldo Cruz: Suicídio: pesquisadores comentam relatório da OMS, que apontou altos índices no mundo, 2014.

15. GONÇALVES LRC, et al. Determinantes espaciais e socioeconômicos do suicídio no Brasil: uma abordagem regional, Brasil, Nova econ. vol.21 n.2 Belo Horizonte May/Aug. 2011.

16. IBGE. Instituto Brasileiro de Geografia e Estatística,2017.

17. LOVISI GM, et al. Análise epidemiológica do suicídio no Brasil entre 1980 e 2006. Rev Bras Psiquiatr. 2009;31(Supl II):S86-93.

18. MACENTE LB. ZANDONADE E. Avaliação da completude do Sistema de informação sobre Mortalidade por suicídio na região Sudeste, Brasil, no período de 1996 a 2007. J Bras Psiquiatr. 2010;59(3):173-181.

19. MACHADO DB. Impacto da desigualdade de renda e de outros determinantes sociais na mortalidade por suicídio no Brasil. Universidade federal da Bahia -UFBA, 2014; $100 \mathrm{p}$.

20. NATARELLI TRP, et al. The impact of homophobia on adolescent health. Escola Anna Nery 19(4) Out-Dez 2015.

21. OLIVEIRA EFA; GARCIA MLT. A política de saúde mental no estado do Espírito Santo Rev. katálysis vol.14 no.1 Florianópolis jan./jun. 2011.

22. PACHECO JS, et al. Tentativa de suicídio em mulheres por queimaduras. Rev. Rene. Fortaleza, v. 11, n. 2, p. 152160, abr./jun.2010.

23. PRATA V; MILANEZ N. Filosofia do suicídio: quando o corpo tem vez [recurso eletrônico] Vitória da Conquista: Labedisco, 141 p. 2016.

24. SANTOS AS, et al. Prevalência de transtornos mentais nas tentativas de suicídio em um hospital de emergência no Rio de Janeiro, Brasil. Cad. Saúde Pública, Rio de Janeiro, 25(9):2064-2074, set, 2009.

25. SCHLÖSSER A; ROSA GFC; MORE CLO. Revisão: Comportamento Suicida ao Longo do Ciclo Vital. Trends in Psychology/ Temas em Psicologia - 2014, Vol. 22, no 133, 1-145.

26. SCHNITMAN G, et al. Taxa de mortalidade por suicídio e indicadores socioeconômicos nas capitais brasileiras. Revista Baiana de Saúde Pública. v.34, n.1, p.44-59 jan./mar. 2010.

27. SINAN. Sistema de informação de agravos notificados.

28. VIDAL CEL, et al. Perfil epidemiológico do suicídio na microrregião de Barbacena, Minas Gerais, no período de 1997 a 2012. Cad. Saúde Colet., 2014, Rio de Janeiro, 22 (2): 158-64.

29. VIDAL CEL; GONTIJO ECDM; LIMA LA. Tentativas de suicídio: fatores prognósticos e estimativa do excesso de mortalidade. Cad. Saúde Pública, Rio de Janeiro, 29(1):175-187, jan, 2013.

30. VIEIRA KFL; SARAIVA ERA; COUTINHO MPL. Entrelaçamentos entre depressão e suicídio segundo os futuros psicólogos. Psico, Porto Alegre, PUCRS, v. 41, n. 2, pp. 176-183, abr./jun. 2010. 